\title{
The Even-Odd and the Isoelectronicity Rules Applied to Single Covalent Bonds in Ionic, Double-Face-Centered Cubic and Diamond-Like Crystals
}

\author{
Geoffroy Auvert ${ }^{1}$, Marine Auvert ${ }^{2}$ \\ ${ }^{1}$ Grenoble Alp University, Grenoble, France \\ ${ }^{2}$ University of Strasbourg, Strasbourg, France \\ Email: Geoffroy.auvert@grenoble-inp.org
}

Received 12 February 2016; accepted 20 March 2016; published 23 March 2016

Copyright (C) 2016 by authors and Scientific Research Publishing Inc.

This work is licensed under the Creative Commons Attribution International License (CC BY). http://creativecommons.org/licenses/by/4.0/

\begin{abstract}
Although atom configuration in crystals is precisely known thanks to imaging techniques, there is no experimental way to know the exact location of bonds or charges. Many different representations have been proposed, yet no theory to unify conceptions. The present paper describes methods to derive bonds and charge location in double-face-centered cubic crystals with 4 and 6 atoms per unit cell using two novel rules introduced in earlier works: the even-odd and the isoelectronicity rules. Both of these rules were previously applied to ions, molecules and some solids, and the even-odd rule was also tested on two covalent crystal structures: centered-cubic and single-face-centered cubic crystals. In the present study, the diamond-like structure was subjected to the isoelectronicity rule in order to derive Zinc-blende structures. Rock-salt-like crystals were derived from each other using both rules. These structures represent together more than 230 different crystals. Findings for these structures are threefold: both rules describe a very sure method to obtain valid single covalent-bonded structures; single covalent structures can be used in every case instead of the classical ionic model; covalent bonds and charges positions do not have any relation with the valence number given in the periodic table.
\end{abstract}

\section{Keywords}

Covalent Bond, Even-Odd, Rule, Single Bond, Chemical Structure, Crystal, Solid, Ionic Crystal, Face-Centered Crystal, Diamond-Like 


\section{Introduction}

In recent papers, the even-odd and the isoelectronicity rules were introduced as a systematic method to precisely predict positions of bonds and charges of atoms in chemical compounds. The rules were successively proven to be effective on ions and molecules [1]-[5], as well as on specific crystal structures [6]. The objective pursued here is to study the possibility to extend the field of application of these rules to other crystal structures.

Crystals are highly organized periodic arrangements extending in the three spatial dimensions [7]. This ordered arrangement of atoms is based on a small 3D pattern, named a unit cell and represented by a small imaginary box. In such a structure, interconnections between atoms are supposed to be either covalent bonds or ionic bonds. First coined in 1919 [8] [9], the term covalent bond describes bonds that are made of electrons pairs. In the case of ionic bonds, however, electrostatic attraction between opposite charges is assumed to hold atoms together. Ionically bonded atoms can form ionic crystals [10], in which charged atoms are simply packed as efficiently as possible [11] without covalent bonds.

More than 200 different forms of unit cells are known [12]. The present paper treats of two isometric cubic crystals: diamond-like and rock-salt-like crystals with covalent bonds. In order to limit the number of crystals exposed here to a reasonable amount, only crystals with mono-element and di-element compounds will be described. Both of these structures are double-face-centered-cubic structures. The diamond-like structure [13] is studied first to test the validity of the isoelectronicity rule with 4 covalent bonds per atom. The rock-salt-like structure [14] is studied in a second phase with both rules applied to atoms with 6 covalent bonds. This paper concludes on the validity of both rules and proposes to dispense with the concept of a classical ionic structure in crystals.

All compounds and structures named in this article derive from the "crystallography open data base" [15]. The software application used to draw crystalline structures with atom positions and atom interconnections is Avogadro V1 [16], completed with GIMP2 to indicate charge positions [17].

\section{Double-Face-Centered Cubic Crystals: Diamond-Like and Rock-Salt-Like.}

There are many different unit cells in crystallography. For the sake of clarity, only one structure is studied in the present article: the double-face-centered cubic structure with 8 atoms per unit cell: the term "double" means the presence of two face-centered structures in the same unit cell. Two structures of this crystal are represented in Figure 1: each unit cell corresponds to a cube with red lines on its borders. Each cube has 8 cell-corners, 6 faces and 12 borders. The atoms are represented by spheres which belong to one or several unit cells: each corneratom belongs by $1 / 8$ in a unit cell, an atom on a border line belongs to $1 / 4$ of a unit cell, a face-centered atom belongs to $1 / 2$ of a unit cell and one atom inside one cell belongs to it.

Figure 1 shows two different "double"-face-centered cubic structures in which atoms of the same color belong to the same single-face-centered structure.

In Figure 1, the first structure is named a diamond-like crystal and the second is named a rock-salt-like crystal.
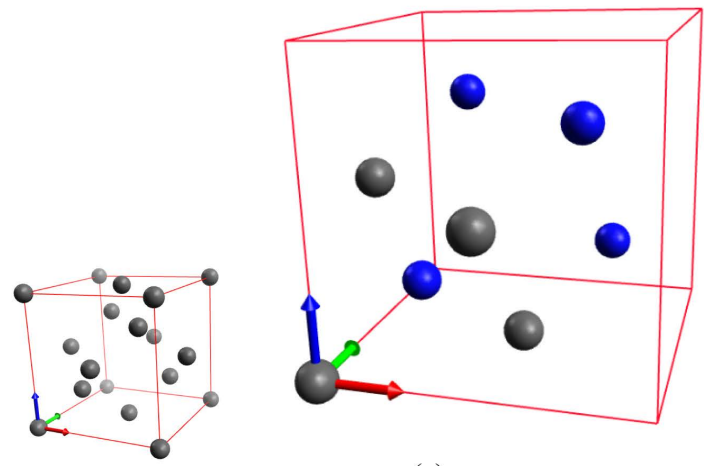

(a)

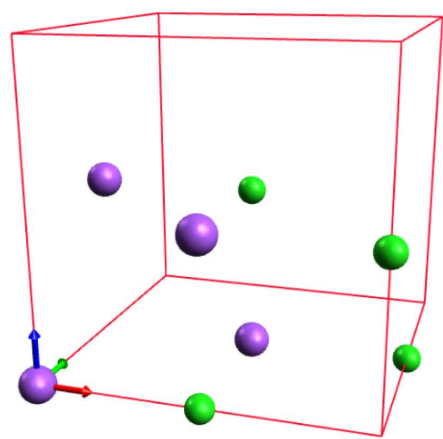

(b)

Figure 1. Two double-face-centered cubic crystals with 8 atoms per unit cell: (a) double-face-centered cubic unit cell with a translation of $(1 / 4,1 / 4,1 / 4)$ from black atoms to blue atoms and (b) double-face-centered cubic unit cell with a translation of $(1 / 2,0,0)$ from purple atoms to green atoms. In (a) and (b), each small cube gives a global view of all the atoms belonging to the corresponding unit cell. 
Both structures are composed of two series of 4 atoms. In Figure 1(a), four black spheres (the first series) are placed in the cube like in a single-face-centered crystal. By duplicating these spheres and applying a $(1 / 4,1 / 4$, 1/4) translation, we obtain the second series. The cube has consequently 8 atoms in the unit cell forming a double-face-centered cubic crystal.

In Figure 1(b), the same construction is achieved with a translation of $(1 / 2,0,0)$ in the horizontal direction. It gives two series of 4 atoms in the unit cell. The first series is purple and the second is green.

Figure 1(a) represent a structure that can be either a diamond-like structure $\mathbf{F} \mathbf{~ d - 3 m ~ [ 1 8 ] ~ o r ~ a ~ z i n c - b l e n d e ~ c r y s - ~}$ tal structures F-4 3m [19]. The unit cell has corner atoms, face-centered atoms and internal atoms but no atom on the edges. The total number of atoms per cell is 8: one corner (8/8), 3 faces (6/2) and 4 internal atoms but no atom in the center of the unit cell. This cubic crystal gives 4 first-neighbors for each atom whatever its position.

Figure 1(b) depicts a structure that can typically be halite, sphalerite or rock-salt-like crystals with a structure reference named $\mathbf{F} \mathbf{~ m - 3 m ~ [ 1 4 ] ~ [ 1 9 ] . ~ T h e ~ u n i t ~ c e l l ~ o f ~ t h i s ~ f i g u r e ~ h a s ~ a ~ c o r n e r ~ a t o m ~ ( 8 / 8 ) , ~} 3$ face-centered atoms $(6 / 2), 3$ atoms on the edges (6/2) and one internal atom (1/1). The total number of atoms per cell is 8 . This cubic crystal gives 6 first-neighbors for each atom whatever its position.

Only these double-face-centered structures composed of two series of single-face-centered configurations, will be used in this paper.

In the following, two rules will be described. The first addresses how to assign charges to atoms and which of them are connected through single covalent bonds. The second rule is a guide to obtain internal coherence when charges and atoms are modified, while the crystal structure remains unchanged.

\section{The Even-Odd Rule for Crystals with Single Covalent Bonds}

In scientific literature, known unit cells are represented with atoms at given positions. Bonds are occasionally shown between atoms in the unit cell but charges are rarely located. When shown, the bonding configuration does not follow a clear rule. A procedure has been recently proposed to draw bonds and to position charges in ions and molecules [5]. This procedure has been tested on crystals [6]. This comparison was successful for a large number of crystals with 8 covalent bonds per atoms. In this paper this rule is described briefly and is applied to cubic crystals with 8 atoms per unit cells and 4 or 6 covalent bonds per atoms.

\subsection{Elements of the Periodic Table and Number of Electrons}

In the periodic table, an element is an atom with a name described by one or two letters and it has an atomic number [20]. The atomic number is equal to the number of positive charges of the nucleus and the number of electrons belonging to that element resulting in a neutral atom. In the periodic table, electrons around the nucleus are not chemically connected to another element.

In this paper, the elements of the periodic table are sorted into two groups: the first group is composed of even-atoms which have an even number of electrons, and the second of odd-atoms which have an odd number of electrons. For instance, carbon is an even-atom with 6 electrons and boron is an odd-atom with 5 electrons.

\subsection{Inner Shell and Covalent Shell for Each Atom in a Solid}

In a solid, the electrons of an atom can be separated in two shells, the inner shell and the covalent shell. The inner shell contains pairs of electrons. In this shell, the number of electrons is subsequently even. The nucleus and the inner shell can be considered as inscribed into a sphere. Around this sphere, several single covalent bonds are presents. They interconnect neighboring atoms and form a covalent shell around each sphere of the solid. To represent a covalent bond, a line is drawn between two spheres in the unit cell. As a covalent bond is composed of an electrons pair, the total number of electrons in a covalent shell is also an even number.

\subsection{Number of Bonds for Each Atom}

In the present article, the number of covalent bonds per atoms is limited to 4 for diamond-like structure [13] [18] and to 6 for rock-salt-like crystals [14] [19]. A single exception will be presented in the discussion.

\subsection{The Even-Odd Rule}

In the even-odd rule, a solid is made out of atoms surrounded by single covalent bonds [5]. This rule described 
earlier [4] is as follow:

- A neutral even-atom must have an even number of covalent bonds;

- A neutral odd-atom must have an odd number of covalent bonds;

- An even-atom with an odd number of bonds must bear a charge $(-)$ or $(+)$;

- An odd-atom with an even number of bonds must bear a charge $(-)$ or $(+)$.

The charge must be considered as including $(-)$ or excluding $(+)$ one electron of the total number of electrons of the atom.

After the constitution of the covalent bonds, both the number of electrons remaining in the inner shell and the total number of electrons in the covalent shell are even.

\subsection{Neutrality of the Unit Cell for 8 Atoms per Unit Cell}

The total charge of a unit cell must be neutral, i.e. all positive charges must be balanced by negative charges. As the unit cell is composed of 8 atoms, the neutrality is obtained by having the same number of positive and negative charges in a single unit cell.

The next rule described is the isoelectronicity rule, which gives the frame for replacing an element with another element without any change in the configuration of the unit cell.

\section{Isoelectronicity Rule in Double-Face-Centered Crystals}

In the present article, cubic unit cells are limited to mono-element and di-element compounds.

In a compound composing a unit cell, any atom can be replaced by another one only according to the isoelectronicity rule [3] described below:

- Original atoms in the solid all have:

- an inner shell with an even number of electrons;

- a covalent shell composed of single covalent bonds;

- a covalent shell number limited (in this paper) to 4 or 6 covalent bonds;

- a charge to compensate the odd number of bonds in an even-atom or the even number of bonds in an odd-atom.

- New atoms have:

- a different name in the periodic table;

- the same covalent bond structure;

- no impact on the unit cell geometry.

- The inner shells of original and new atoms are different:

- the new element has a different number of electrons pairs in its inner shell;

- a different charge to compensate the change of the number of electrons and to follow the even-odd rule as described just above.

- Conservation of the neutrality of the unit cell:

- the number of charged atoms must be an even number to allow the neutrality;

- each charge must be compensated by an opposite charge of another first or second neighbor atom in the cell.

We will name di-element compounds when their components belong to the periodic columns. For instance, $\mathrm{MgO}$ is a $2-6$ di-element compound, i.e. $\mathrm{Mg}$ belongs to column 2 and $\mathrm{O}$ belongs to column 6 of the periodic table.

Although both rules seem to be restrictive, they are in the following successfully applied to well-known double-face-centered cubic crystals: The first one is a diamond-like crystal.

\section{Double-Face-Centered Cubic Crystals: Diamond-Like Crystals}

Many famous crystals have diamond-like structures. This structure, shown in Figure 2, contains 8 atoms per unit cell at the same positions than in Figure 1(a). In the diamond-like structure, carbon atoms are represented as black spheres with four black lines around each atom representing four covalent bonds for each carbon atom.

The drawing of this structure is in agreement with the even-odd rule: The even-atom of carbon does not bear a charge due to the even number of bonds [1].

As shown in the right column in Figure 2, this structure exists with 6 different atoms [15] (one is a 4 - 4 di- 


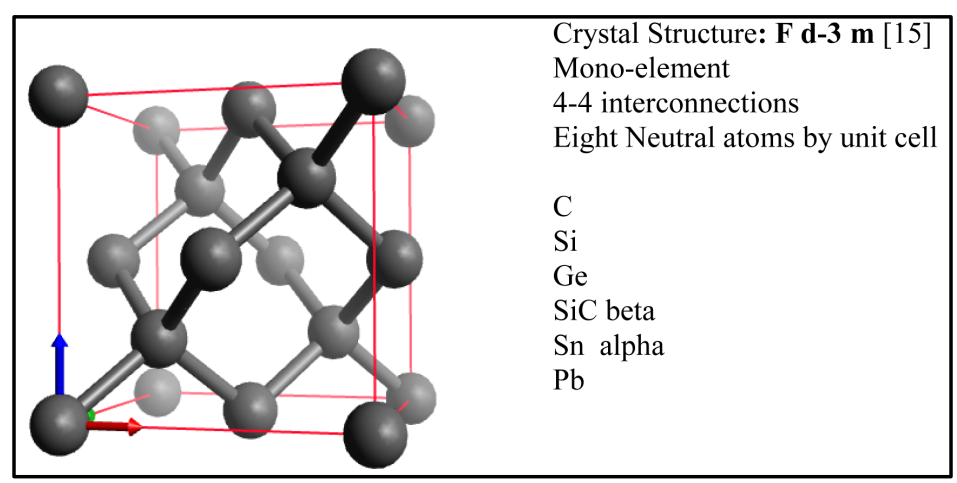

Figure 2. Double-face-centered cubic crystal with eight carbon atoms and sixteen covalent bonds in a unit cell. In right column, a list of atoms names having this $\mathbf{F} \mathbf{d}-\mathbf{3} \mathbf{~ m}$ structure [15].

element compound).

In the following, the isoelectronicity rule is used to replace each pairs of atoms of Figure 2, by a di-elementcompound of odd-atoms without any structure modification.

\section{Double-Face-Centered Cubic Crystals: First Charged Zinc-Blende}

A zinc-blende structure is a cubic crystal in which atoms have the same positions in the unit cell as in Figure 2. The difference lies in the type of atoms composing the crystal. The unit cell is composed of a di-elements compound repeated four times. The structure shown in Figure 3 is obtained by applying the isoelectronicity rule described earlier on the diamond structure. The neutral carbon atoms of the first single-face-centered series are replaced by a positively charged element from column 5 in the periodic table. To compensate the positive charge, the second series of carbon is replaced by negatively charged elements from column 3 .

In the resulting construction (Figure 3), the grey series of atoms form the first single-face-centered structure and the brown series is the second single-face-centered series. The unit cell with 8 atoms is therefore a doubleface-centered cubic structure.

By comparing Figure 2 and Figure 3, all 8 atoms are at the same location and interconnections are at the same position. Moreover, grey atoms missing one electron, have the same number of electrons in their covalent shell. In the same way, brown atoms, with an extra electron, all have the same number of electrons in their covalent shell. This confirms the ability of the isoelectronicity rule to have no modification in the covalent structure of the unit cell.

The zinc-blende structure of Figure 3, also named sphalerite has an F-4 3 m structure.

Each atom in Figure 3 is bonded to other atoms of the di-element compound through four covalent bonds and each bond links two opposite charges, resulting in the overall neutrality of the unit cell. As a consequence, the validity of the even-odd rule is maintained. Additionally, as detailed in the right-hand column of Figure 3, the

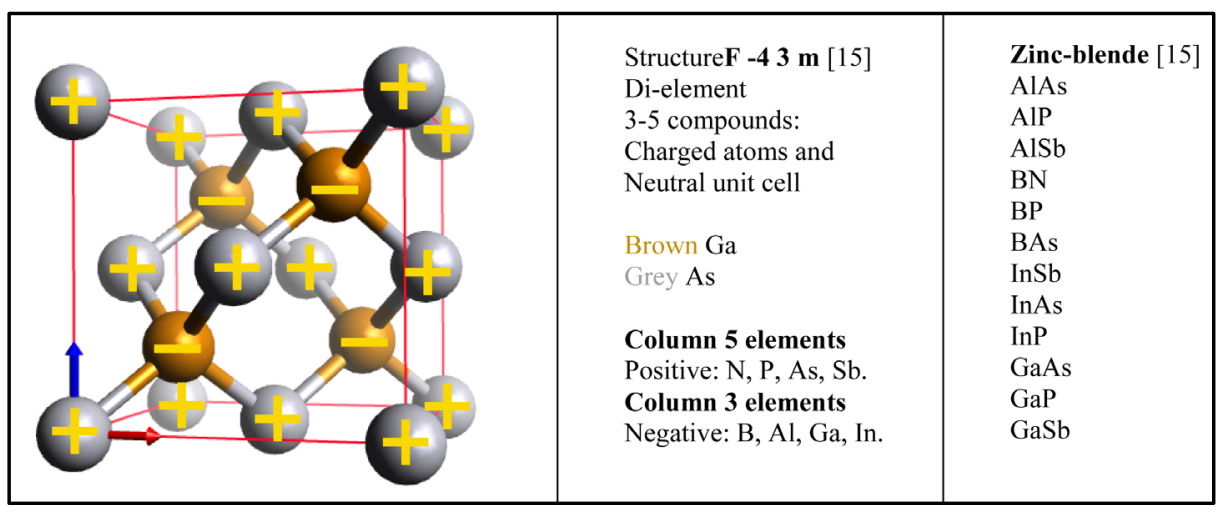

Figure 3. Double-face-centered cubic crystal with 3 - 5 di-element compounds of odd-atoms. 
isoelectronicity rule is applicable by imposing both 4 atoms of column 3: Boron, aluminum, gallium and indium with a negative charge, and the 4 others from column 5: Phosphorus, arsenic, antimony and bismuth with a positive charge.

The next double-face-centered cubic structure is with neutral atoms of 2-6 di-element compounds.

\section{Double-Face-Centered Cubic Crystals: Neutral Zinc-Blende}

Another configuration of zinc-blende crystals is composed of neutral di-element compounds repeated four times i.e. with even-atoms. This configuration of di-element even-atoms has a diamond-like structure but the atoms belong respectively to columns 2 and 6 in the periodic table. One example of these is the well-known di-element compound $\mathrm{ZnSe}$, as represented in Figure 4. The dark grey zinc atoms form the first series (see chapter 2) of the single-face-centered structure. The red selenium atoms are inside the unit cell and compose the second single-face-centered series. In this structure, possible atoms from column 2B are zinc, cadmium and mercury whereas beryllium is from column 2. Possible elements from column 6 are sulfur, selenium and tellurium. Di-element compounds listed in the right column of Figure 4, have all a zinc-blende structure with neutral atoms and are in agreement with the even-odd rule: They are even-atoms with 4 covalent bonds.

The next structure is obtained by applying an isoelectronic transformation of these neutral compounds into charged di-element compounds.

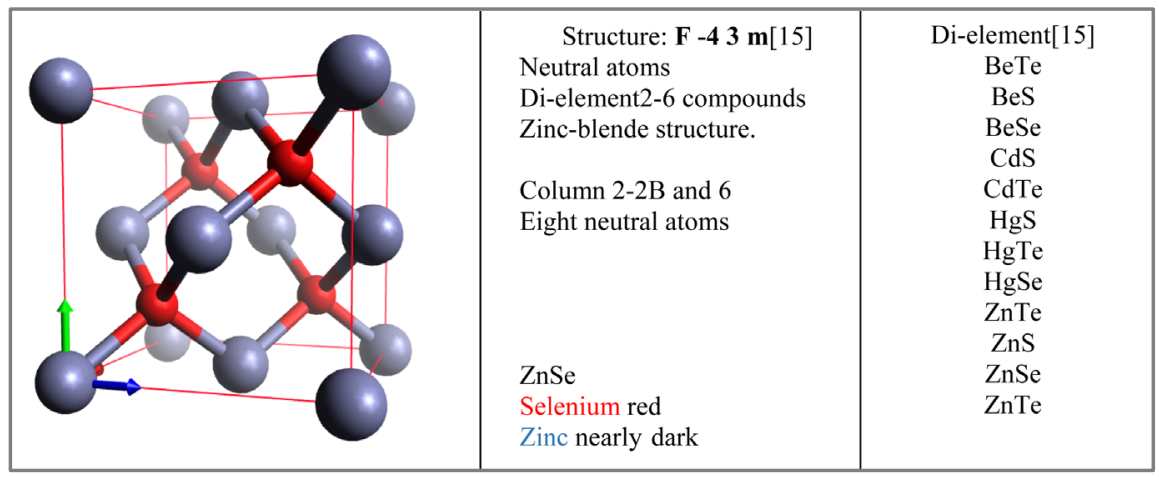

Figure 4. Neutral even-atoms with four covalent bonds in zinc-blende structure. Grey atoms from column 2 or $2 \mathrm{~B}$ and red atoms are from column 6.

\section{Double-Face-Centered Cubic Crystals: Second Charged Zinc-Blende}

The structure of Figure 4 is composed of neutral di-element compounds. They can be replaced by charged elements using the isoelectronicity rule described earlier. Using Figure 4 as a reference, the isoelectronicity result is displayed in Figure 5. Grey elements from column 2 are replaced by a negatively charged yellow element

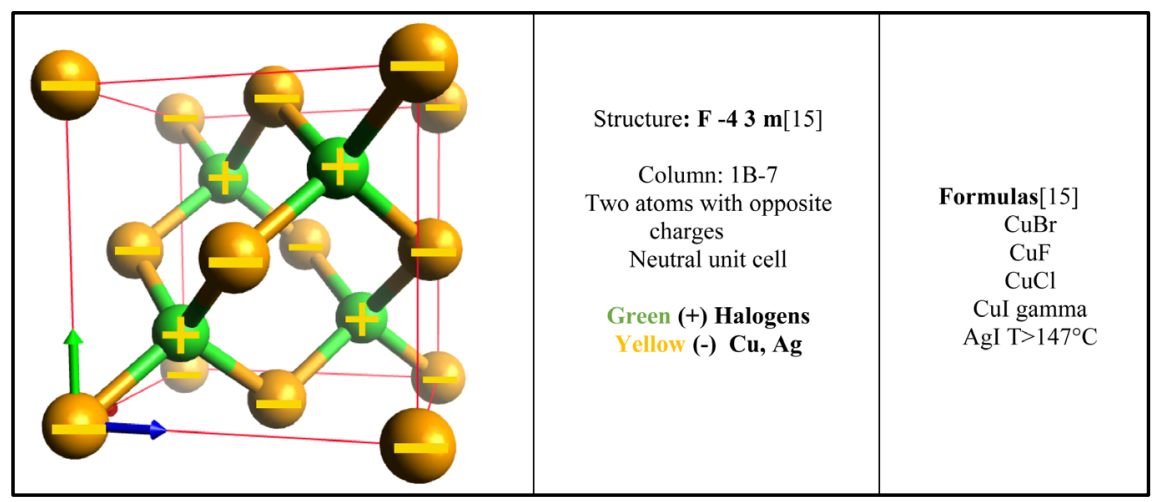

Figure 5. Double-face-centered cubic crystal with charged atoms: 1 - 7 di-element compounds. The neutral reference is with $2-6$ di-element compounds and the isoelectronicity transformation is a charged $1(-)-7(+)$ di-element compounds. 
from column 1 (or 1B) while red elements from column 6 are replaced by a positively charged green element from column 7. After this transformation, the number of bonds is unchanged and covalent bonds remain the same. The unit cell stays overall neutral with four negative and four positive charges. Finally, this structure still agrees with the even-odd rule.

A well-known example of a di-element compound with this structure is $\mathrm{CuCl}$. In Figure 5, the negatively charged copper atoms are displayed in yellow and the positively charged chloride atoms are displayed in green. In such a structure, halogen atoms, like $\mathrm{Cl}(+)$, behave like sulfur atoms.

\section{Double-Face-Centered Cubic Crystals: Half-Charged Zinc-Blende}

In this chapter, the isoelectronicity rule concerns the second series (see Figure 1(a)) of the double-face-centered cubic structure.

Another possibility to build a different structure with a diamond-like structure of Figure 2 is first to conserve neutral atoms of the first single-face-centered cubic series and to build the second single-face-centered cubic series with odd-atoms but with a slightly different isoelectronicity rule. The result appears in Figure 6. All four blue even-atoms still bear no charge while among the four brown atoms, two are negatively and two are positively charged. The atoms positions and the covalent bonded geometry are both conserved.

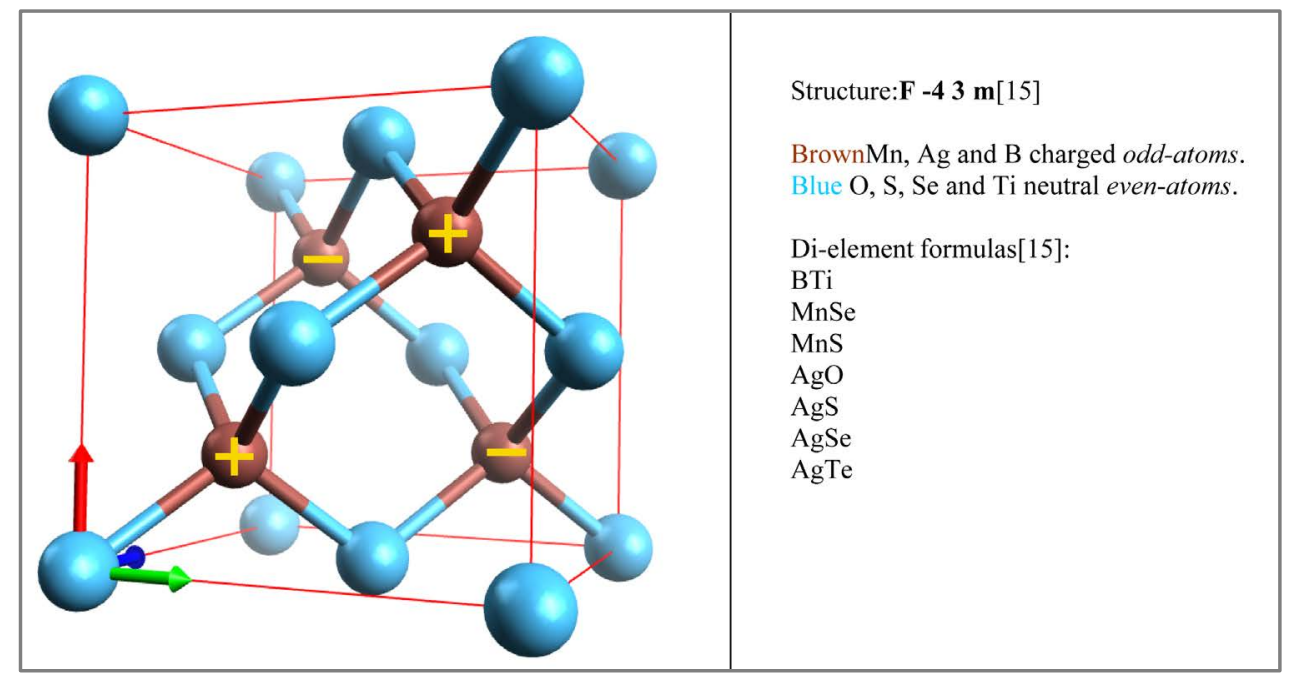

Figure 6. Double-face-centered cubic crystal with in blue color: neutral atoms for first face-centered series, and in brown color: charged atoms for the second (translated) face-centered series.

In paragraphs 5 to 9, all di-element compounds have an $\mathbf{F ~ d - 3 m}$ or an $\mathbf{F - 4} \mathbf{3} \mathbf{~ m}$ structure and are in agreement with the even-odd rule. Covalent bonds and atoms positions are indeed conserved also when applying the isoelectronicity rule. The use of both rules, even-odd and isoelectronicity, is very helpful for the constructions of these structures.

In this first part of the article, the authors have observed that none of the cubic structures shown have any relation with the value of the valence number obtained from the periodic table. The authors may propose that the valence number is not needed to analyze bonding configuration in crystal structures.

Another observation is that atoms in these solids are neutral or bear a single charge. For the authors, this seems to be an implicit consequence of both the even-odd rule and the compatibility with the isoelectronicity rule.

In the second part of the article, rock-salt-like crystals are studied. These structures are also double-face-centered cubic crystals in which atoms lie at different positions compared to diamond-like crystals.

\section{Double-Face-Centered Cubic Crystals: Neutral Rock-Salt-Like}

Rock-salt-like structures are adopted by a large number of di-element compounds including many alkali-halides [14] [15]. Di-element oxides such as $\mathrm{MgO}$ take also this shape. A procedure to build this double-face-centered structure is displayed in Figure 7: two single-face-centered cubic structures (fccs) are needed. The first one in 
Figure 7(a), is the standard representation of the single-fccs with 4 atoms per unit cell and the second one in Figure 7(b), is also a single-fccs with 4 atoms per unit cell but with a translation of $(1 / 2,0,0)$ from the first one. The superposition is shown in Figure 7(c). The result is a double-fccs with 8 atoms per unit cell and each atom has 6 first neighbors. These six neighbors are shown in a color differing of that from the first atoms.

In these di-element compounds, even-atoms are uncharged.

Figure 8 shows known atoms as in Figure 7(c) with their interconnections. Figure 8(a) depicts how six single covalent bonds are drawn around the center atom of the unit cell. For a di-element compound, (one green and one red sphere), the single covalent bonds only link different elements. In Figure 8(b), the even-odd pro-

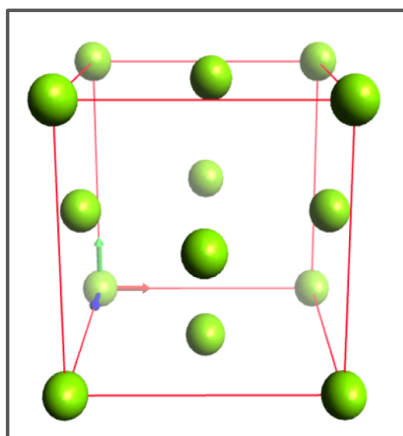

Single-face-centered cubic

(a)

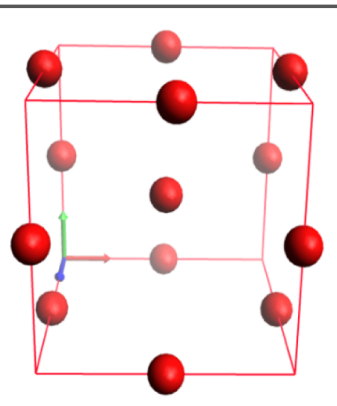

Translated $(1 / 2,0,0)$ or $(1 / 2,1 / 2,1 / 2)$ of a single-face-centered cubic

(b)

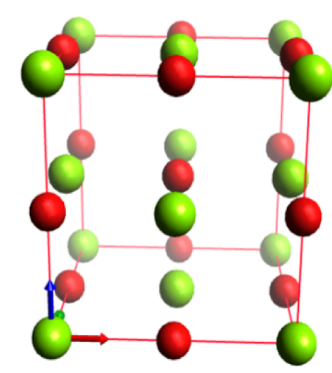

double-face-centered cubic

(c)

Figure 7. Two single and one double-face-centered cubic crystal: (a) classical single-face-centered cubic structure with green atoms; (b) second single-face-centered cubic with red atoms is translated of $(1 / 2,0,0)$ from (a); (c) superposed both single-face-centered crystal structures giving a double-face-centered crystal structure. A red atom has 6 green first neighbors (and inversely).

\begin{tabular}{|c|c|c|c|}
\hline (a) & Di-element even-atoms & $\begin{array}{l}\text { Main group 2-6 } \\
\text { Uncharged elements } \\
6 \text { bonds per atoms } \\
\text { Red Column } 6 \\
\text { Green Column } 2\end{array}$ & $\begin{array}{l}\text { Structure:Fm-3m[15] } \\
\text { Alkaline earth metal } \\
\\
\mathrm{Ba}(\mathrm{O}, \mathrm{S}, \mathrm{Se}, \mathrm{Te}) \\
\mathrm{Ca}(\mathrm{O}, \mathrm{S}, \mathrm{Se}, \mathrm{Te}) \mathrm{Mg}(\mathrm{O}, \mathrm{S}, \mathrm{Se}) \\
\mathrm{Sr}(\mathrm{O}, \mathrm{S}, \mathrm{Se}, \mathrm{Te})\end{array}$ \\
\hline (b) & Di-element even-atoms & $\begin{array}{l}\text { Metals of column } 6 \\
\text { Red column } 4 \text { or } 6 \\
\text { Brown metal } \\
\mathrm{Cd}(\mathrm{O}, \mathrm{Te}) \\
\mathrm{Ce}(\mathrm{S}, \mathrm{Se}, \mathrm{Te}) \\
\mathrm{DyTe} \\
\mathrm{ErTe} \\
\mathrm{FeO} \\
\mathrm{GdSe} \\
\mathrm{HfC} \\
\mathrm{HgTe} \\
\mathrm{Nd}(\mathrm{C}, \mathrm{O}, \mathrm{S}, \mathrm{Se}, \mathrm{Te}) \\
\mathrm{NiO}\end{array}$ & $\begin{array}{l}\text { Structure : Fm-3m } \\
{[\mathbf{1 5}]} \\
\mathrm{Pb}(\mathrm{S}, \mathrm{Se}, \mathrm{Te}) \\
\mathrm{SnSe}, \mathrm{SnTe} \\
\mathrm{Sm}(\mathrm{O}, \mathrm{Se}, \mathrm{Te}) \\
\mathrm{TaC} \\
\mathrm{ThC}, \mathrm{ThS}, \mathrm{ThSe} \\
\mathrm{TiC}, \mathrm{TiO} \\
\mathrm{U}(\mathrm{O}, \mathrm{S}, \mathrm{Se}, \mathrm{Te}) \\
\mathrm{Yb}(\mathrm{O}, \mathrm{Se}, \mathrm{Te}) \\
\mathrm{ZnTe} \\
\mathrm{Zr}(\mathrm{C}, \mathrm{O}, \mathrm{S})\end{array}$ \\
\hline
\end{tabular}

Figure 8. Double-face-centered cubic crystal with di-element compounds of even-atoms: (a) six single covalent bonds for the center atom; (b) all single bonded connections of the unit cell are drawn. 
cedure is applied to draw bonds of each other atoms. In the right of this figure, the list of di-element is given as long as they have even-atoms and where no bonds interconnect two spheres with the same color, i.e. the same element.

Nearly sixteen different neutral di-element compounds of main group elements (see right column in Figure $8(a))$ are known to take this configuration. Each even-atom is in agreement with the even-odd rule: no charge is needed and an even number of single covalent bonds are drawn.

The same structure is obtained in Figure 8(b) for twenty transition metals (some are mono-oxide or monotelluride) in which transition metals are even-atoms.

In the next paragraph, for odd-atoms in a di-element compound, charges have to be allocated.

\section{Double-Face-Centered Cubic Crystals: Charged Rock-Salt Like}

Without any change in the above rock-salt-like structure, the even-odd rule teaches how some neutral evenatoms can be replaced by charged odd-atoms. There are two possible results of this transformation. The first one is shown in Figure 9, and the second in Figure 10. In the first transformation, oxide atoms remain while atoms from column 2 are replaced by transition-metal odd-atoms bearing a charge, positive or negative, in agreement with the proposed even-odd rule.

In Figure 9, green even-atoms are the same than the red even-atoms in Figure 8(b). The brown atoms have been replaced by an odd-atom and must bear a charge to keep the even number of bonds. To preserve the overall neutrality of the unit cell, half of the odd-atoms should be positively charged and the other half negatively charged. Charges are assigned in planes, as shown in Figure 9. A list of di-element compounds crystallizing into this charged structure is given in the right column of Figure 9.

The second structure is obtained when all atoms of Figure 8 are replaced by odd-atoms. This transformation from a neutral structure is obtained by replacing an even-atom of column 6 into a charged halogen atom of column 7 and in the same way; the other even-atoms are replaced by a charged odd-atoms. Some of them can belong to column 1 or 1B in the periodic table. This is shown in Figure 10.

Halogen atoms with a positive charge are: Fluor, Chlorine, Bromine and Iodine. The negatively charged atoms from column 1 are Sodium, potassium, rubidium and cesium or transition odd-atoms (like Silver (47)), which belong to column $1 \mathrm{~B}$.

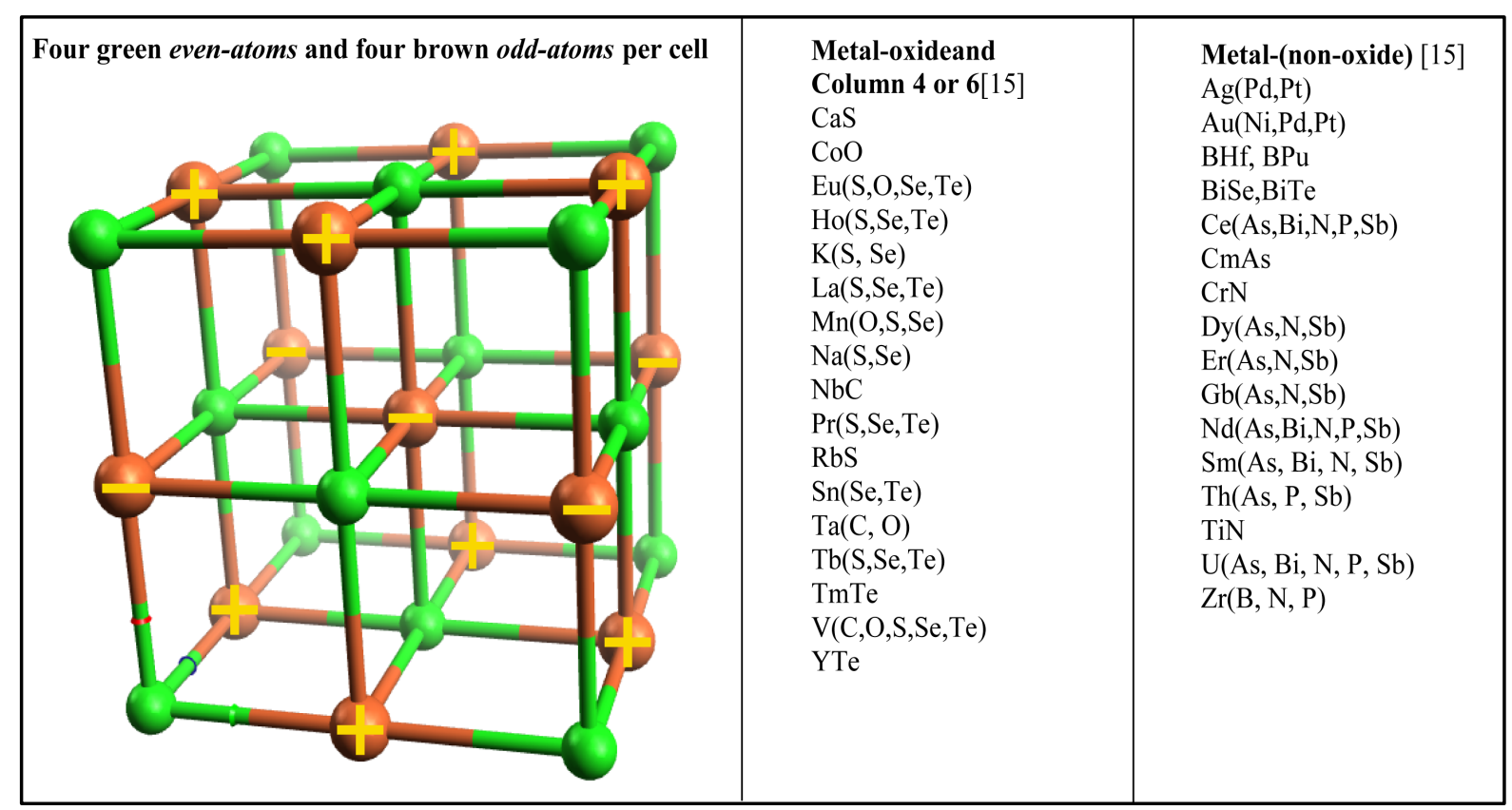

Figure 9. Double-face-centered cubic crystals with 6 single covalent bonds per atom: green color for neutral even-atoms and brown color for charged odd-atoms. 


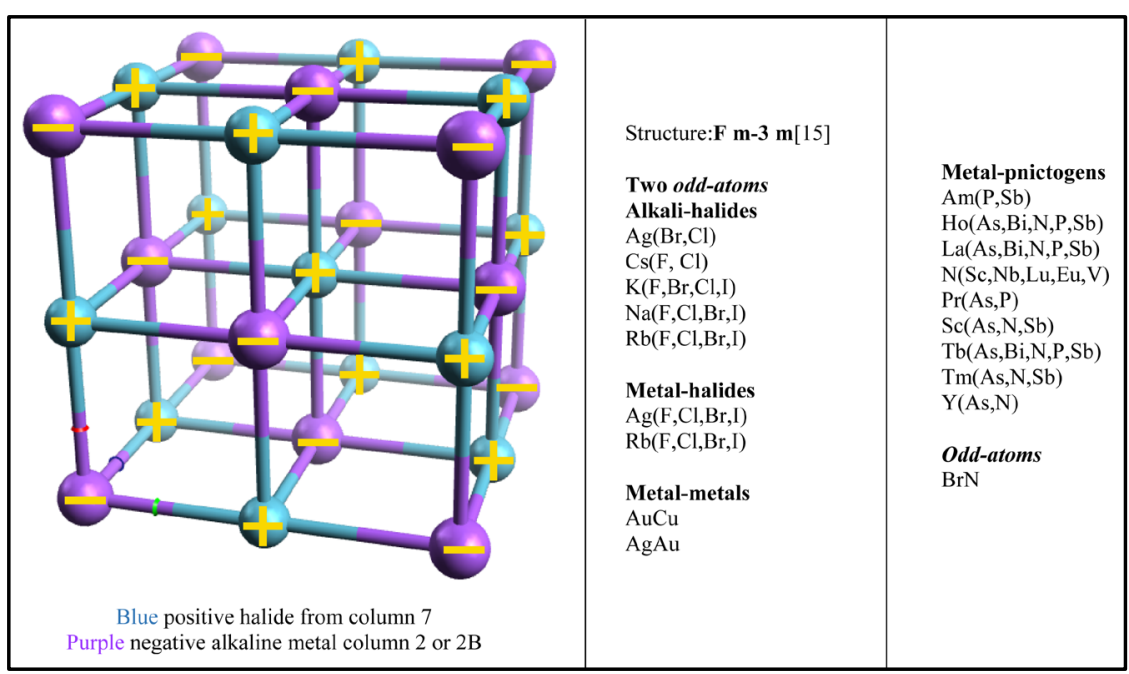

Figure 10. Charged rock-salt structure where two elements of the neutral rock-salt structure are replaced. Halogen atoms are positively charged and most metal atoms with a negative charge are from column 1 or $1 \mathrm{~B}$ in the periodic table.

In Figures 8-10, all di-element compounds adopting the $\mathbf{F m - 3 m}$ structure [15] are compatible with the evenodd rule.

We now discuss particular structures.

\section{Discussion}

\section{- Cubic crystals}

More than 230 configurations in double-face-centered cubic structures are mentioned in the present article. This number is wide and allows the authors to open interesting discussions on the validity of the rules.

\section{- The validity of the even-odd rule}

The validity of the even-odd rule was recently tested on single-face-centered crystals [6]. For the authors, the previous article reports a valid test to extend the even-odd rule from molecules to crystals with 4 atoms per unit cell. In the present article, the success with 8 atoms in cubic unit cells is without ambiguity and the rule is validated on double-face-centered cubic crystal with 6 covalent bonds per atom.

\section{- The validity of the isoelectronicity rule}

The isoelectronicity rule was formerly applied to molecules and ions [3]. In this article, the rule is applied to neutral 4 - 4 structures, to obtain charged 3 - 5 structures, and to neutral 2 - 6 structures, to create charged 1 - 7 structures. 30 crystals could be included thanks to this rule. This is great enough to consider the validity of the isoelectronicity rule for crystals.

As a consequence, this rule seems applicable to every structure: ions, molecules and crystals.

\section{- The structure of $\mathrm{NaCl}$ crystals and the ionic configuration}

Figure 8(b) reports that the di-element $\mathrm{MgS}$, a 2-6 compound, has an $\mathbf{F m - 3} \mathbf{~ m}$ structure with neutral atoms and 6 bonds per atoms. This is also shown in Figure 11(a) where red atoms are sulfur even-atoms and grey atoms are magnesium even-atoms. By following the isoelectronicity rule, even-atoms are replaced by odd-atoms: $\mathrm{Mg}$ by $\mathrm{Na}(-)$ and $\mathrm{S}$ by $\mathrm{Cl}(+)$. The result is drawn in Figure 11(b).

In the same way, another $\mathrm{NaCl}$ crystal can be deduced from an argon crystal and compared to that of Figure 11(b).

In the periodic table, Argon is an even-atom in column 8. Argon can crystallize into a mono-element evenatom single-face-centered cubic crystal as shown in Figure 12(a), with 8 covalent bonds per atom [6]. Following the isoelectronicity rule, 4 argon atoms are replaced by $\mathrm{Na}(+)$ and the four others replaced by $\mathrm{Cl}(-)$. The result is shown in Figure 12(b).

Let us compare both obtained structure (Figure 11(b) and Figure 12(b)): the first is $\mathrm{Na}(-)$ and $\mathrm{Cl}(+)$ with 8 covalent bonds and the second one gives $\mathrm{Na}(+)$ and $\mathrm{Cl}(-)$ with 6 bonds. Therefore, two different structures are obtained with the isoelectronicity rule, showing that it is possible to build two $\mathrm{NaCl}$ crystal structures where 


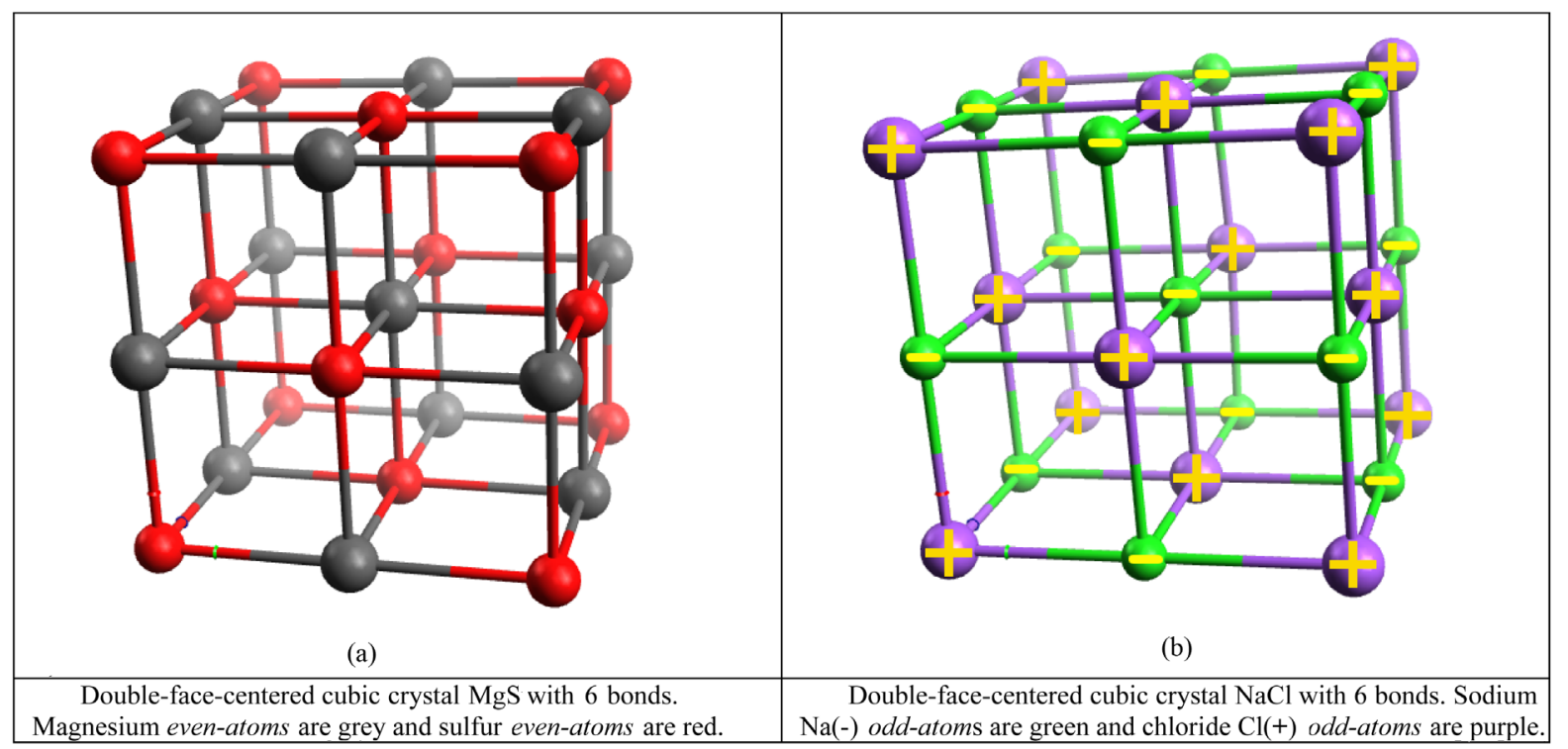

Figure 11. Two isoelectronic double-face-centered cubic crystals: (a) MgS crystal with 6 bonds per atoms and in (b) $\mathrm{Na}(-) \mathrm{Cl}(+)$ cubic crystal with 6 bonds.

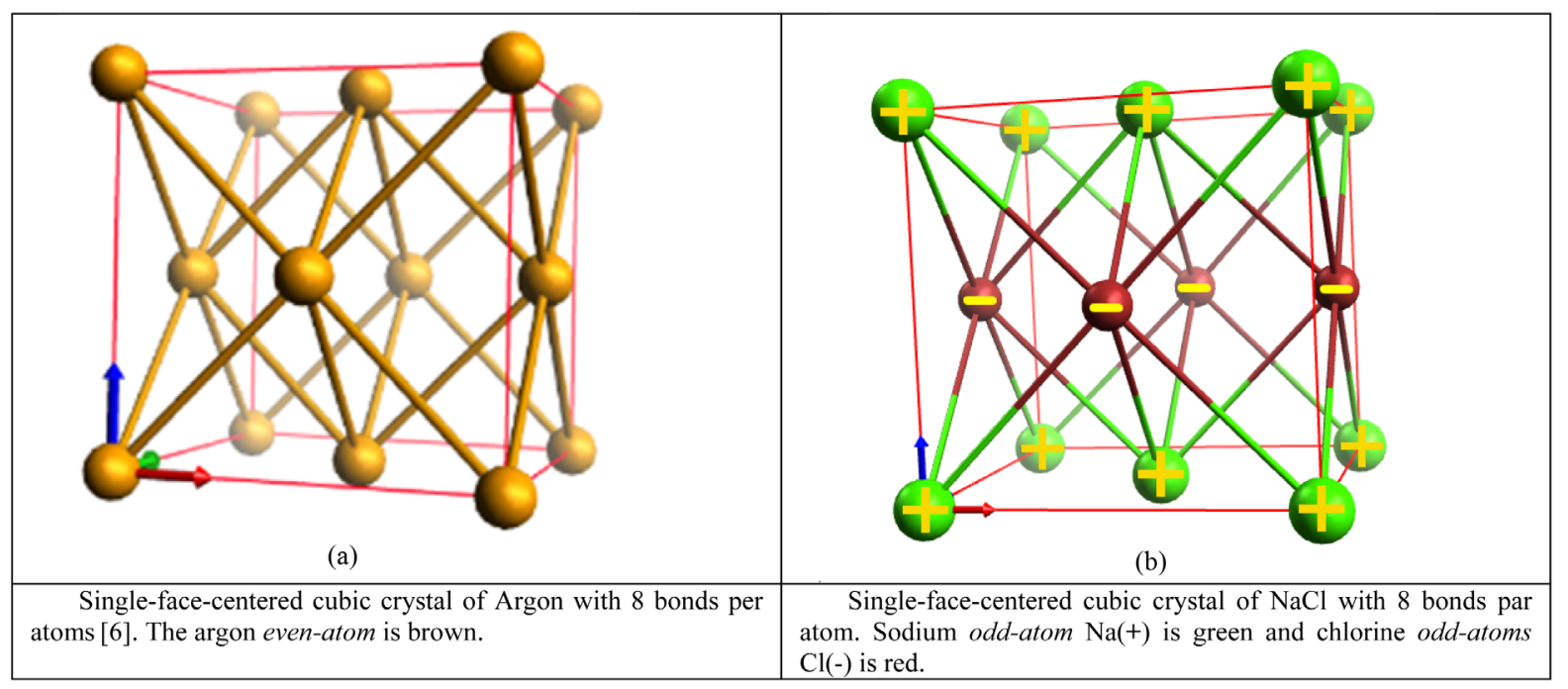

Figure 12. Two isoelectronic single-face-centered cubic crystals: (a) argon crystal with 8 bonds per atoms [6] and in (b) $\mathrm{Na}(+) \mathrm{Cl}(-)$ cubic crystal with 8 bonds per atom.

atoms of sodium and chlorine are covalently bonded. For the authors, this finding could imply that the usual ionic structure [11] is dispensable to explain salt-like crystals. The main consequence of this article is thus that a unique binding model based on single covalent bonds may be sufficient for cubic crystals.

\section{- Number of covalent bonds and the valence number}

We have in the present paper studied firstly crystals with four covalent bonds per atom and secondly crystals with six covalent bonds. By comparing the valence number of the elements used, the authors have found that several valence numbers are not related to the solid structures. It is therefore straightforward to propose that the number of covalent bonds in a crystal does not have a correspondence with the valence number of the element. Even an oxygen atom, which is known to erect only two bonds, has six bonds in CaO neutral doubleface-centered crystals (Figure 8(a)).

In the same way, the normal valence number of 4 for carbon has no influence. This is shown in the second crystalline structures where the carbon element appears in 10 di-element compounds with 6 bonds per carbon. 
These compounds shows that elements connected to the carbon are lanthanides or transition metals. Their column number in the periodic table is far away on the left side and below the carbon position. The authors venture the hypothesis that elements at this location in the periodic table have the ability to open a space around the carbon atom and allow the latter to involve all of its six electrons into covalent bonds.

\section{- Structure names}

The first diamond-like structure described is $\mathbf{F ~ d - 3 ~} \mathbf{m}$ (Figure 3). The next three are F-4 $\mathbf{3} \mathbf{~ m}$ (Figures 4-6). In other words, existing theory proposes 2 structures names while this article highlights 4 different structures. It may be interesting to improve on the classical nomenclature to create appropriate names for the 4 structures studied above.

\section{- Comparing $\mathrm{BeO}, \mathrm{MgO}$ and $\mathrm{CaO}$}

$\mathrm{Be}, \mathrm{Mg}$ and $\mathrm{Ca}$ belong to the same column of the periodic table. One would naturally expect them to adopt the same crystal structure. $\mathrm{MgO}$ and $\mathrm{CaO}$ crystallize into identical rock-salt-like crystals with 6 covalent bonds. It is surprisingly not the case for $\mathrm{BeO}$ which adopt a hexagonal wurtzite structure, with four covalent bonds.

The total number of electrons in beryllium is equal to 4 and this limits the number of covalent bonds it can establish. This atom cannot use two extra electrons from its neighbors to add two covalent bonds i.e. to have a beryllium atom with 6 covalent bonds. For the authors, the main consequence of this is that an atom in a solid cannot bear several charges, such as $2(+)$ or $2(-)$. The same hypothesis is imposed in the isoelectronicity rule to add or subtract only one charge. This supposition was previously used [5].

\section{- Particularity for Lanthanides elements}

In the periodic table, the lanthanides group consists of 15 elements on the same row. Lanthanides elements erect 6 bonds in the double-face-centered crystal (Figure 9). This seems to indicate that the number of bonds does not depend on the number of electrons of the lanthanides which can be either odd-atoms or even-atoms. To explain this singularity, the authors suggest that all lanthanides might actually have nearly the same external electrons repartition in their covalent shells. In other words, all even-lanthanides and all odd-lanthanides respectively have the same number of electrons in their covalent shells. The other electrons are captured by their inner-shells.

\section{Conclusion}

Two rules are the starting point of this study: the even-odd rule gives a procedure to draw single covalent bonds in solids, and the isoelectronicity rule gives a procedure to transform selected atoms inside a solid without changing the bonding configuration. The first rule confirms its ability to construct two types of double-facecentered cubic crystals. The second rule, previously applied to molecules, is now confirmed for diamond-like crystals. Thanks to the isoelectronicity rule, neutral crystals of $4-4$ and $2-6$ compounds can be successfully transformed into crystals of $3-5$ and $1-7$ compounds with charged atoms. By analyzing the large number of compounds in various structures, it has been observed that the valence number given by the periodic table has no influence on the structures studied in this article. Applying both rules also result in finding two possible covalently bonded $\mathrm{NaCl}$ crystals, a cubic structure with 4 bonds $(\mathrm{Na}(+) \mathrm{Cl}(-))$ and of 6 bonds $(\mathrm{Na}(-) \mathrm{Cl}(+))$. The usual ionic bonded structure seems thus unnecessary. Finally, both rules can assign single charges on specific atoms without ambiguity in every case named here. The corollary is that elements cannot bear more than one charge, being positive or negative.

\section{References}

[1] Auvert, G. (2014) Improvement of the Lewis-Abegg-Octet Rule Using an "Even-Odd" Rule in Chemical Structural Formulas: Application to Hypo and Hyper-Valences of Stable Uncharged Gaseous Single-Bonded Molecules with Main Group Elements. Open Journal of Physical Chemistry, 4, 60-66. http://dx.doi.org/10.4236/ojpc.2014.42009

[2] Auvert, G. (2014) Chemical Structural Formulas of Single-Bonded Ions Using the "Even-Odd" Rule Encompassing Lewis's Octet Rule: Application to Position of Single-Charge and Electron-Pairs in Hypo- and Hyper-Valent Ions with Main Group Elements. Open Journal of Physical Chemistry, 4, 67-72. http://dx.doi.org/10.4236/ojpc.2014.42010

[3] Auvert, G. (2014) Coherence of the Even-Odd Rule with an Effective-Valence Isoelectronicity Rule for Chemical Structural Formulas: Application to Known and Unknown Single Covalent-Bonded Compounds. Open Journal of Physical Chemistry, 4, 126-133. http://dx.doi.org/10.4236/ojpc.2014.43015

[4] Auvert, G. (2014) The Even-Odd Rule on Single Covalent-Bonded Structural Formulas as a Modification of Classical 
Structural Formulas of Multiple-Bonded Ions and Molecules. Open Journal of Physical Chemistry, 4, 173-184. http://dx.doi.org/10.4236/ojpc.2014.44020

[5] Auvert, G. (2015) How the Even-Odd Rule, by Defining Electrons Pairs and Charge Positions, Can Be Used as a Substitute to the Langmuir-Octet Rule in Understanding Interconnections between Atoms in Ions and Molecules. Open Journal of Physical Chemistry, 5, 28-38. http://dx.doi.org/10.4236/ojpc.2015.52004

[6] Auvert, G. and Auvert, M. (2015) Chemical Bonds between Charged Atoms in the Even-Odd Rule and a Limitation to Eight Covalent Bonds per Atom in Centered-Cubic and Single Face-Centered-Cubic Crystals. Open Journal of Physical Chemistry, 5, 93-105. http://dx.doi.org/10.4236/ojpc.2015.54010

[7] https://en.wikipedia.org/wiki/Crystal

[8] Lewis, G.N. (1916) The Atom and the Molecule. Journal of the American Chemical Society, 38, 762-785. http://dx.doi.org/10.1021/ja02261a002

[9] Langmuir, I. (1919) The Arrangement of Electrons in Atoms and Molecules. Journal of the American Chemical Society, 41, 868-934. http://dx.doi.org/10.1021/ja02227a002

[10] Pauling, L. (1929) The Principles Determining the Structure of Complex Ionic Crystals. Journal of the American Chemical Society, 51, 1010-1026. http://dx.doi.org/10.1021/ja01379a006

[11] https://en.wikipedia.org/wiki/Ionic_compound

[12] https://en.wikipedia.org/wiki/Crystal_structure

[13] https://en.wikipedia.org/wiki/Diamond

[14] https://en.wikipedia.org/wiki/Cubic_crystal_system

[15] Downs, R.T. and Hall-Wallace, M. (2003) The American Mineralogist Crystal Structure Database. American Mineralogist, 88, 247-250. http://www.crystallography.net/search.html

[16] Hanwell, M.D., Curtis, D.E., Lonie, D.C., Vandermeersch, T., Zurek, E. and Hutchison, G.R. (2012) Avogadro: An Advanced Semantic Chemical Editor, Visualization, and Analysis Platform. Journal of Cheminformatics, 4, 17.

[17] https://en.wikipedia.org/wiki/GIMP

[18] https://en.wikipedia.org/wiki/Diamond_cubic

[19] https://en.wikipedia.org/wiki/Sphalerite

[20] https://en.wikipedia.org/wiki/Periodic table 